\title{
Statistical Validation of Cardiovascular Digital Biomarkers Towards Monitoring the Cardiac Risk in COPD: A Lyfas Case Study
}

\author{
Subhagata Chattopadhyay ${ }^{*}{ }^{(\mathbb{C})}$, Rupam Das \\ Acculi Labs Pvt. Ltd, R. R. Nagar, Bangalore, Karnataka, 560098, India \\ Email: subhagata.chattopadhyay2017@gmail.com
}

Received: 17 November 2021; Revised: 21 December 2021; Accepted: 29 December 2021

\begin{abstract}
Background: Mobile health (mHealth) is gaining popularity due to its pervasiveness. Lyfas is a smartphonebased optical biomarker instrument catering to mHealth. It captures the Pulse Rate Variability (PRV) and its associated digital biomarkers from the index finger capillary circulation using the principle of arterial photoplethysmography. PRV surrogates for the Cardiovascular Autonomic Modulation (CvAM) and provides a snapshot of psychophysiological homeostasis of the body. Objective: The paper investigates the roles of (a) physiological factors, e.g., Age, Duration of illness, Heart Rate (HR), Respiration Rate (RR), $\mathrm{SpO}_{2}$ level, and (b) popular digital biomarkers, such as $\mathrm{SDNN}, \mathrm{LF} /$

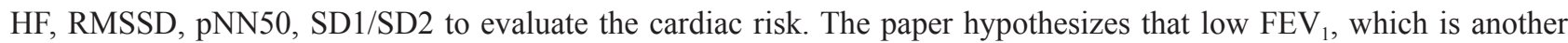
physiological factor, plays a critical role in defining such risk. Method: A total of 50 males and females each, suffering from Chronic Obstructive Pulmonary Disease (COPD) took the Lyfas test after appropriate ethical measures. Data, thus collected by Lyfas had been statistically analyzed using histogram plots and Kolmogorov-Smirnov test for normality check, Pearson's Correlations (PC) to measure the strength of associations, and linear regressions to test the goodness of fit of the model. Results: Positive PCs are noted between (a) RMSSD and SDNN ('very high'-females: 0.86 and males: 0.91), (b) pNN50 and RMSSD (PC: moderate 0.46), (c) pNN50 and SDNN (PC: moderate 0.44), (d) Duration of illness and Age ('high'-females: 0.71 and males: 0.77), and (e) Age and RR ('high'-females: 0.67, males: 0.53). Negative PC is noted between (a) LF/HF and $\mathrm{FEV}_{1}$ ('moderately high'-males 0.42) and (b) LF/HF and $\mathrm{SpO}_{2}$ ('moderately high'-males 0.30 ). Although the $R_{2}$ values are not so encouraging (most are $<0.5$ ), yet, the models are statistically significant ( $p$-values 0.0336; CI 95\%). Conclusion: The paper concludes that Lyfas may be used to predict the cardiac risk in COPD patients based on the $\mathrm{LF} / \mathrm{HF}$ values correlated to $\mathrm{SpO}_{2}$ and $\mathrm{FEV}_{1}$ levels.
\end{abstract}

Keywords: Lyfas, Pulse Rate Variability (PRV), Cardiac Autonomic Modulation (CAM), Cardiovascular Autonomic Modulation (CvAM), digital biomarkers, home healthcare, physiological factors, COPD

\section{Introduction}

Smartphone-based mobile health or $m$ Health is gaining popularity in today's digital healthcare. The advantages lie in its (i) pervasiveness, (ii) non-invasive nature, (iii) economical, and (iv) personalized approach. The growth of mHealth is proportional to the steady increment of smartphone users across the globe and the reduced cost of mobile data and the expansion in Internet access. The in-built optical sensor, LED light, high data processing speed, and

Copyright (C2022 Subhagata Chattopadhyay, et al

DOI: https://doi.org/10.37256/aie.3120221252

This is an open-access article distributed under a CC BY license

(Creative Commons Attribution 4.0 International License)

https://creativecommons.org/licenses/by/4.0/ 
higher storage capacity of the cloud stores are available by default in the phones and pose to be the key advantages of smartphone usage as the much-personalized healthcare tools. From the business perspective, the mHealth market size is $\$ 46,048$ million in 2019 to $\$ 230,419$ million by 2027 [1]. Signal processing algorithms, such as Fast Fourier Transform, Autoregressive Spectral Estimation Techniques, Wavelet transform, Lplacean transform, Curvelet transform, Hilbert transform, and so forth are most commonly used to retrieve medically important information from the electrical signals obtained from various structures, such as heart, brain, eyes, ears, etc [2]. Lyfas is a biomedical application that runs on smartphones with Android version 7 or more operating systems. It uses the principle of Reflection Arterial PhotoPlethysmography (RAPPG) in capturing the Capillary Pulse Rate Variability (CPRV) and its associated digital biomarkers (together called Cardiovascular digital biomarker, which deputes for the Heart Rate Variability (HRV) and its associated digital biomarkers [3]. It is important to note that the HRV and its associated digital biomarkers provide the physiological snapshot of the Cardiac Autonomic Modulation (CAM), i.e., modulation of the heart by the Sympathetic and Parasympathetic Nervous Systems that fall under the Autonomic Nervous System (ANS) to maintain the cardiac homeostasis. In addition to that, CPRV provides the snapshot of Cardiovascular Autonomic Modulation (CvAM), i.e., providing the snapshots of vascular health (e.g., arterial stiffness). Therefore, by evaluating the digital biomarkers using CPRV with Lyfas, the cardiovascular health of an individual can be assessed [4].

Chronic Obstructive Pulmonary Disease (COPD) is a heterogeneous chronic inflammatory disease of the respiratory system causing 3.23 million global deaths and is the third leading cause of death and over $80 \%$ of deaths happen in the low-and-middle-income nations [5]. It is characterized by persistent and progressive respiratory difficulties, especially exhalation (evident by lower $\mathrm{FEV}_{1}$ values), and excessive mucous production in the respiratory passage. As the condition progresses (i.e., $\mathrm{FEV}_{1}$ values diminishes), it poses a high risk of (i) right ventricular failure, clinically called Cor Pulmonale [6], and finally, (ii) Bi-Ventricular Failure [7], where the respiratory distress is even worse characterized by orthopnoea (severe respiratory distress while lying, which is relieved with sitting posture) [8], (iii) increased blood-tinged mucous production due to rupture of the bronchial vessels as a consequence of strenuous breathing, (iv) deep cyanosis due to lack of oxygen in the tissues, (v) cardiac edema leading to fluid accumulation in the dependent part of the body, (vi) paroxysmal nocturnal dyspnea (also known as the cardiac COPD), and finally (vii) respiratory muscle failure [9]. Therefore, $\mathrm{FEV}_{1}$ is a critical pathophysiological parameter to assess the cardiac risk in COPD patients [10]. Other important cardiovascular cascades of COPD are tachycardia due to the increased demand for oxygen in the body, low pulse pressure due to reduced stroke volume, and the rise in the diastolic blood pressure owing to generalized vasoconstriction [11].

The objective of the study is to examine how various physiological factors and digital biomarkers, captured by Lyfas, can explain the plausible cardiac risk of COPD in adults from its validation perspective. Hence, in this work, Optical biomarkers are assigned as the risk indicators, while $\mathrm{FEV}_{1}$ has been the target variable, which is a critical parameter to assume the risk of heart failure in COPD cases. The novelty of the study lies with the observation that the state of COPD can be assessed pervasively, i.e., from anywhere at any time, non-invasively, at a much lower cost, in a personalized manner using Lyfas.

\section{Material and method}

This section is divided into five parts as follows.

\subsection{Ethical compliance}

a. The study protocol was approved by the Vagas Institutional Ethics Committee review board (No. ECR/1181/Inst/ KA 2019, dated 30-01-2020).

b. Signed informed consents of all participants' have been taken according to the declaration of Helsinki by the research team prior test.

\subsection{Duration of study}

This cross-sectional study had been performed from April 2021 to July 2021 in Indian cities. 


\subsection{Factors: their physiological significance and the normal Lyfas values}

a) Cardiovascular digital biomarkers [12-13] are captured from the index finger's peripheral CPRV with Lyfas by utilizing the principle of RAPPG. These biomarkers can be classified as follows. It is worth noting that in this study, only the following five biomarkers have been considered due to their domain specificity:

a. Time-domain components-

i. $S D N N$ (milliseconds) denotes the standard deviation of NN intervals. It refers to the grade of cardiac risk as < 40 high risks, 40-100 moderate risks, $>100$ no risks. The risk attributes to sympathetic dominance.

ii. $p N N 50(\%)$ denotes the percent of NN intervals $>50$ milliseconds. A value $<20 \%$ indicates cardiac risk due to sympathetic dominance.

iii. RMSSD (milliseconds) denotes the root mean square of RR or NN interval difference. A value $<54$ indicates health risk and refers to sympathetic dominance.

b. Frequency-domain components-

i. $L F / H F$ (ratio) denotes the sympathovagal balance, respectively. A value $>2$ refers to cardiac risk due to sympathetic dominance.

c. Non-linear measure-

i. SD1/SD2 (ratio) denotes the ratio of Poincare plot standard deviation perpendicular to the line of identity and denotes the sympathovagal balance. The Lyfas normal range is 1-2.5. Value $>2.5$ poses to be risky for the heart owing to sympathetic dominance.

b) Physiological factors:

a. Age $(A)$ in years, when the Lyfas test has been performed.

b. Duration of illness $(D)$, in years, i.e., the period of illness before the Lyfas test has been taken.

c. Heart Rate (HR, measured in beats per minute or bpm) is the most important vital sign of the body. The normal range is $60-100 \mathrm{bpm}$. A value $<60$ refers to bradycardia and indicates of high vagal tone, while values $>100$ denote tachycardia and are indicative of sympathetic drive [14].

d. Respiratory Rate ( $R R$, which is the number of respirations per minute or rpm) is another important measure of the vital sign of the body. The normal range is $12-16 \mathrm{rpm}$ at rest. In COPD it is $12-20 \mathrm{rpm}$, while $>25$ is considered as an exacerbation [14].

e. Oxygen saturation or $\mathrm{SpO}_{2}$ (expressed in \%) and is a measure of peripheral $\mathrm{O}_{2}$ saturation and is an important indicator for the necessity of $\mathrm{O}_{2}$ administration. In COPD cases the target $\mathrm{SpO}_{2}$ is $88-92 \%$, while in healthy adults it is $95 \%$ and above [15].

f. Body Mass Index (BMI) is a measure of the healthy state of the body and is a ratio of height and weight. It is an important physiological parameter for any metabolic disorder. In a normal adult, it is 18.5-24.9, BMI over 24.9 is considered overweight, and above 30 is obese [16].

g. Forced Expiratory Volume in one second $\left(F E V_{l}\right)$ is an important metric of underlying airflow obstruction. Nonacute cases or mild $\mathrm{COPD}$ where $\mathrm{FEV}_{1}$ is $>80 \%$ of predicted, i.e., no obvious airflow obstruction, moderate COPD where $\mathrm{FEV}_{1}$ is $50-80 \%$ predicted value, i.e., moderate airflow obstruction), and $<50 \%$ indicates severe obstruction [17].

Hence, sympathetic dominance that poses risks of bi-ventricular failure with the progression of COPD needs to be monitored clinically. It is worth noting that, an experienced Interventional Cardiologist and a Pulmonologist have been consulted to conduct this study, starting from the recruitment of the patients to clinical validation of results using Lyfas. This study hypothesizes that in the case of COPD, the physiological signature of the sympathetic dominance can be captured by evaluating the digital biomarkers although ANS tries to compensate it with a parasympathetic buffer.

\subsection{Recruitment of the subjects (total $=100 ; M=50, F=50$ )}

a) Inclusion criteria

a. Adults without any history of diabetes, hypertension, thyroid disorders, chronic kidney diseases, and any other comorbidities.

b. Non-acute cases (mild COPD where $\mathrm{FEV}_{1}$ is $>80 \%$ ) at the time of the test.

c. $\mathrm{SpO}_{2}>90 \%$, i.e., less hypoxemia ) at the time of the test.

d. Not under any medication or on occasional local beta-stimulants and corticosteroids (i.e., just for emergency 
use).

e. No history of admission in hospital with respiratory distress or severe COPD or COVID-19 complications.

f. No history of associated Hypertension, Diabetes Mellitus, Kidney diseases, or any metabolic syndrome, which may affect the CvAM.

\subsection{Lyfas test}

1. FILLING THE PATIENT DETAILS

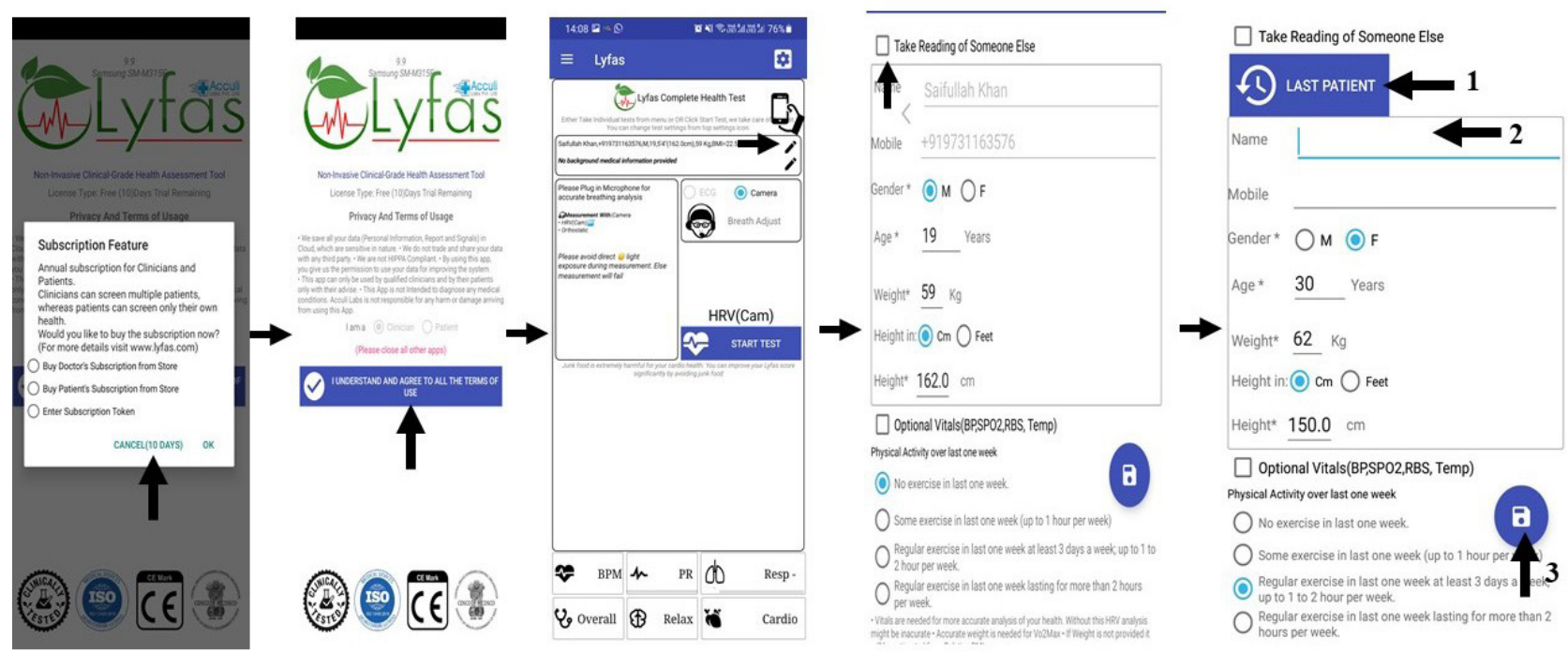

\section{TAKING THE HRV TEST (120 seconds)}

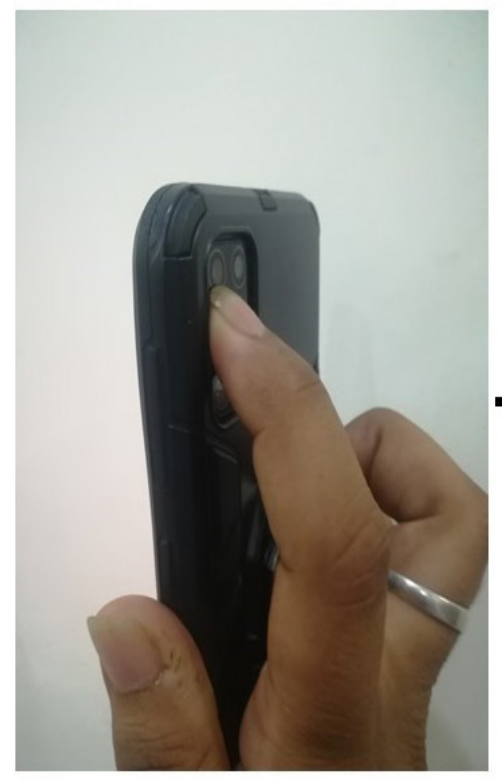

Keep the right index finger on the primary camera

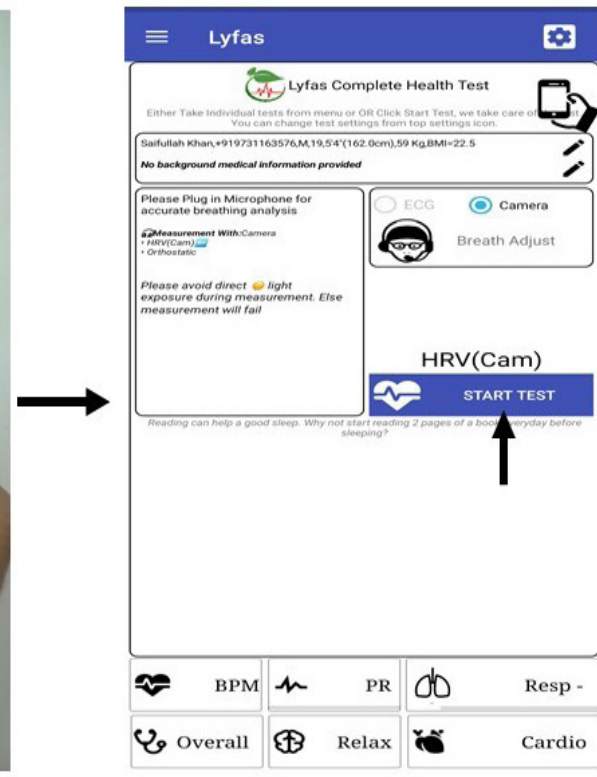

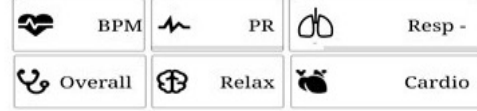

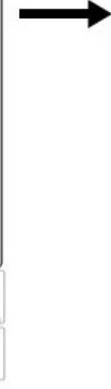

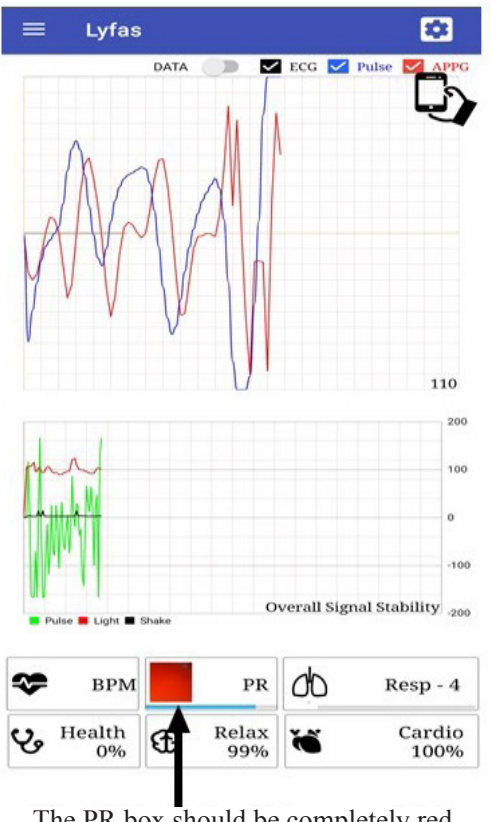

The PR box should be completely red 
3. TAKING THE ORTHOSTATIC TEST (60 seconds)

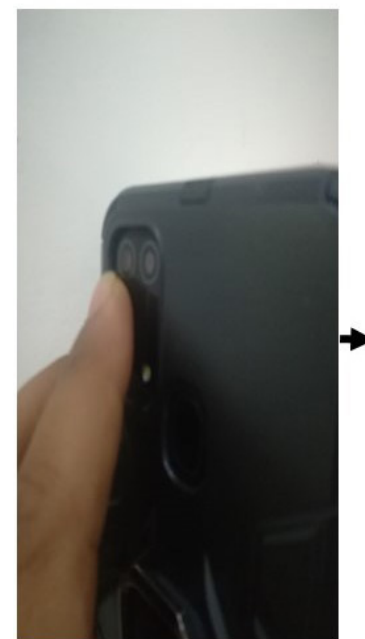

Keep the left index finger on the primary camera

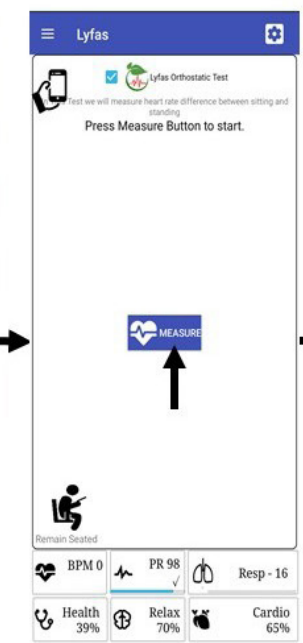

Remain Seated

\section{西}

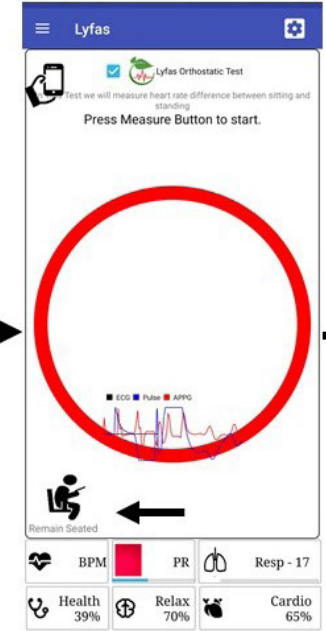

Remain Seated for 15 seconds

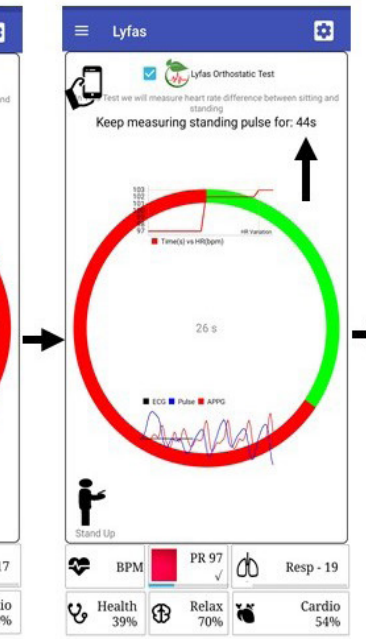

Stand up for 45 seconds
E Lyfas

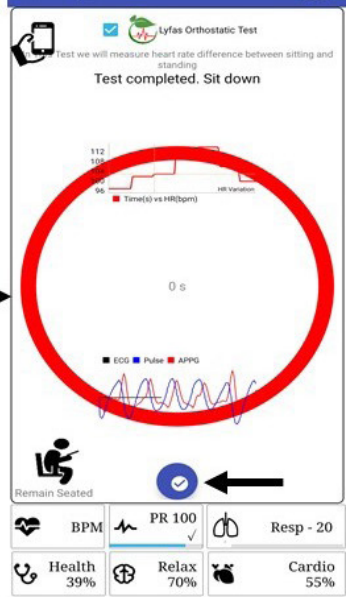

Test Completed

4. REPORT
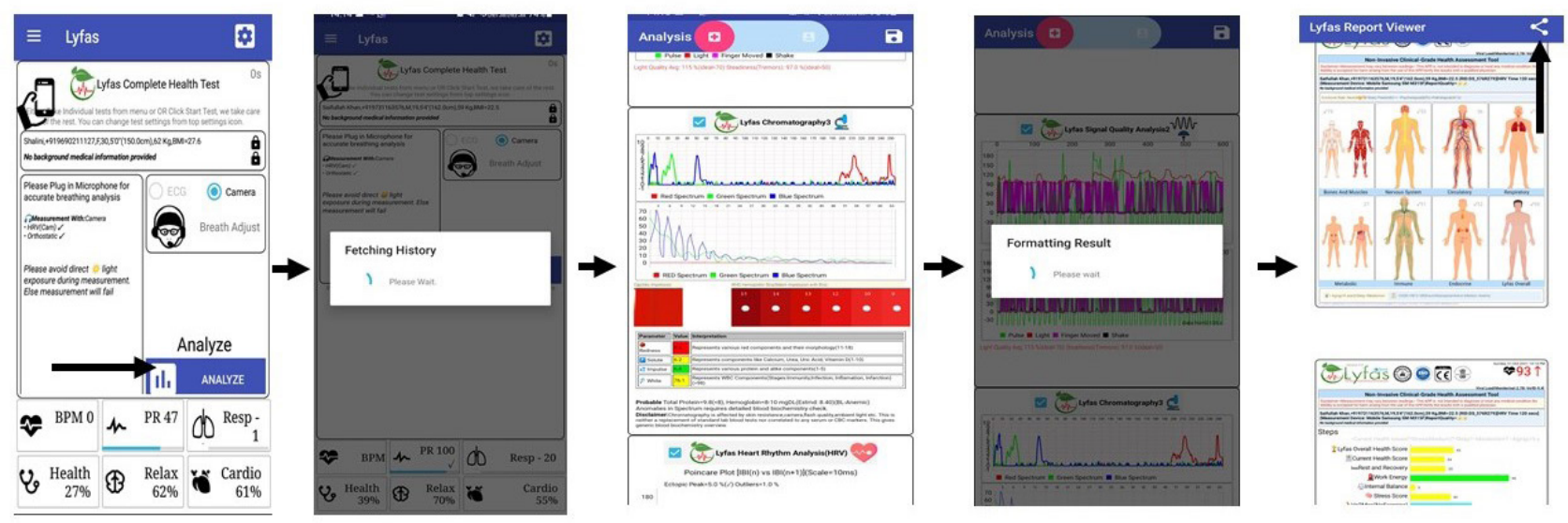

Figure 1. procedural snapshot of the Lyfas test and the analytics

a) Duration: 5 minutes per test

b) Test-time: twice daily-7 am and 7 pm for 7 days per case. Hence there are 6 cardiovascular parameters (HR,

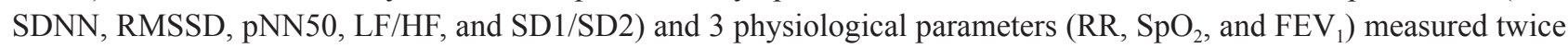
a day for 7 days give total a total of 98 readings for each parameter. In this paper, the mean of the readings for each factor is considered for further analysis. Procedures (Figure 1):

a. Filling in the patient details.

b. Taking the HRV test. Lyfas does the PRV assessment, which surrogates for the HRV test.

c. Taking the orthostatic test, and finally.

d. Report generation.

Digital biomarker scores, thus captured using Lyfas, are then analyzed along with other physiological factors, such as (i) Duration of illness, (ii) Age of the subjects, (iii) HR, (iv) RR, (v) $\mathrm{SpO}_{2}$, (vi) $\mathrm{FEV}_{1}$, and (vii) BMI. 


\subsection{Statistical analysis}

a) Data distribution check (variable-wise Histogram plots), where 'normally' distributed data fits under a uniform bell-shaped or Gaussian type of curve.

b) Kolmogorov-Smirnov test (KST) is a non-parametric test for a null hypothesis as the distribution of a variable is normal, else an alternate hypothesis is satisfied. It has also been conducted to test the normality check, variable-wise, for males and females. A high KST value and low p-value refer to non-normally distributed data. Alongside, the KST, Skewness, and Kurtosis of each variable have also been measured to note the positive or negative deviations from the normal bell-shaped distribution and whether the data has a heavier tail than that of the normal probability distribution, respectively.

c) Descriptive statistics include the mean, maximum value, minimum value, median, and standard deviation calculation for each variable gives the information of data dispersion.

d) Statistical validation.

i. Pearson's Correlation Coefficient (PC) between any two factors is the measure of either a positive, negative, or non-correlation [19]. These values are expressed between-1 (most negative correlation) to +1 (most positive correlation) [20]. All the above-mentioned factors are considered for the PC study. In this work, it is important to note that for any correlation value $<0.1$ has been regarded as trivial or 'no correlation', $0.1-0.25$ as 'low correlation', $>0.25-0.5$ as 'moderate correlation', $>0.5-0.75$ as 'high correlation', and $\geq 0.75$ as 'very high correlation'. Such correlations are applicable for both the negative and positive sides. In this study, the authors have considered the inter-class correlation values from $1^{\text {st }}$ rank i.e., the 'very high' correlation till 'moderate' correlation, whether it is positive or negative. Finally, the $p$-values of the notable PCs are estimated.

ii. Linear regressions, factor-wise have been conducted to see how much ( $\mathrm{R}^{2}$ values) one factor could predict the other. $\mathrm{R}^{2}$ values close to 1.0 are considered to be the high predicting capacity of a factor by the other factor.

Statistical analysis and related coding have been done using Python 3.8 preloaded with NumPy, SciPy, ScikitLearn, Matplotlib, Seaborn, and Pandas packages, run on Windows 10 pro OS with IntelCore processor and $8 G B$ RAM.

\section{Results}

In this section, experimental results are shown. In the next section, the findings are discussed in detail. Before the results are shown, for an understanding of the matrix, sample 'original' data can be found in Tables 1 and 2, where the duration and the age are in years.

Table 1. Sample data of females

\begin{tabular}{ccccccccccccc}
\hline No. & Duration & $\mathrm{Age}$ & $\mathrm{BMI}$ & $\mathrm{HR}$ & $\mathrm{RR}$ & $\mathrm{SpO}_{2}$ & $\mathrm{pNN} 50$ & $\mathrm{LF} / \mathrm{HF}$ & $\mathrm{SD} 1 / \mathrm{SD} 2$ & $\mathrm{RMSSD}$ & $\mathrm{SDNN}$ & $\mathrm{FEV}_{1}$ \\
\hline 1 & 5 & 32 & 33.2 & 128 & 15 & 92 & 34 & 2.2 & 0.79 & 87.5 & 58.3 & 78 \\
2 & 23 & 49 & 30.8 & 115 & 17 & 92 & 42 & 0.7 & 1.15 & 55.8 & 44.2 & 67 \\
3 & 8 & 29 & 28.4 & 104 & 18 & 93 & 25 & 1.3 & 0.37 & 78.3 & 50.2 & 68 \\
4 & 7 & 29 & 28.4 & 115 & 14 & 95 & 46 & 1.3 & 1.13 & 71.4 & 49 & 59 \\
5 & 22 & 59 & 30.4 & 104 & 15 & 92 & 89 & 0.6 & 1.22 & 54.7 & 41.3 & 56 \\
6 & 33 & 55 & 25.6 & 119 & 16 & 91 & 10 & 2.3 & 1.09 & 86.6 & 57.2 & 58 \\
7 & 14 & 38 & 36.2 & 106 & 18 & 91 & 31 & 1.4 & 2 & 46.5 & 41.8 & 56 \\
8 & 12 & 48 & 28.7 & 114 & 21 & 90 & 111 & 1 & 3.64 & 51.8 & 36.9 & 59 \\
9 & 29 & 54 & 27.8 & 103 & 18 & 90 & 50 & 0.8 & 1.19 & 54.5 & 46.5 & 60 \\
\hline
\end{tabular}


Table 2. Sample data of males

\begin{tabular}{ccccccccccccc}
\hline No. & Duration & $\mathrm{Age}$ & $\mathrm{BMI}$ & $\mathrm{HR}$ & $\mathrm{RR}$ & $\mathrm{SpO}_{2}$ & $\mathrm{pNN} 50$ & $\mathrm{LF} / \mathrm{HF}$ & $\mathrm{SD} 1 / \mathrm{SD} 2$ & $\mathrm{RMSSD}$ & $\mathrm{SDNN}$ & $\mathrm{FEV}_{1}$ \\
\hline 1 & 4 & 41 & 36.7 & 101 & 16 & 93 & 33 & 1.6 & 0.9 & 65.7 & 50.2 & 55 \\
2 & 2 & 30 & 37.5 & 119 & 15 & 92 & 31 & 0.8 & 1.09 & 70 & 59.5 & 78 \\
3 & 5 & 37 & 32.1 & 108 & 16 & 94 & 49 & 1.2 & 4.14 & 63.9 & 46.9 & 68 \\
4 & 7 & 23 & 41 & 110 & 17 & 95 & 17 & 2 & 1.16 & 40.6 & 31.9 & 56 \\
5 & 12 & 23 & 36.2 & 112 & 18 & 96 & 17 & 0.8 & 1.17 & 44.6 & 46.9 & 81 \\
6 & 14 & 23 & 35.6 & 124 & 17 & 97 & 19 & 0.8 & 1.07 & 56 & 47.9 & 80 \\
7 & 7 & 25 & 30.5 & 102 & 14 & 91 & 92 & 0.9 & 2.05 & 43.8 & 30.9 & 79 \\
8 & 38 & 65 & 36.2 & 106 & 20 & 90 & 12 & 1.9 & 1.55 & 81.1 & 51.8 & 60 \\
9 & 4 & 41 & 36.7 & 101 & 16 & 93 & 33 & 1.6 & 0.9 & 65.7 & 50.2 & 55 \\
\hline
\end{tabular}

a) Data distribution can be seen in the histograms plots (Figure 2).

(a)
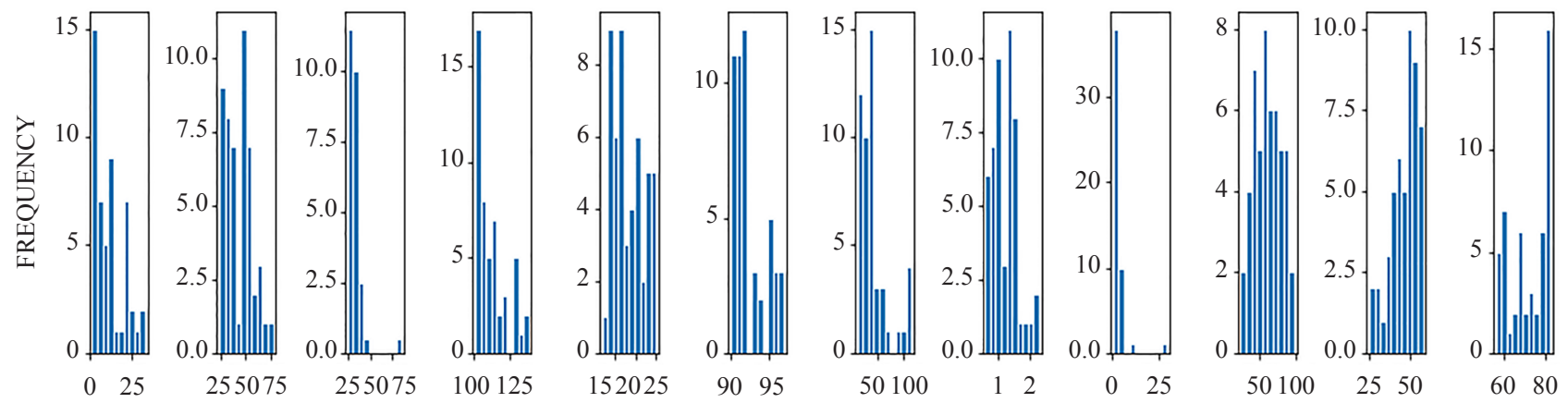

DURATION

BMI

HR

$\mathrm{RR}$

$\mathrm{SpO}_{2}$

RMSSD SDNN

$\mathrm{FEV}_{1}$

(b)
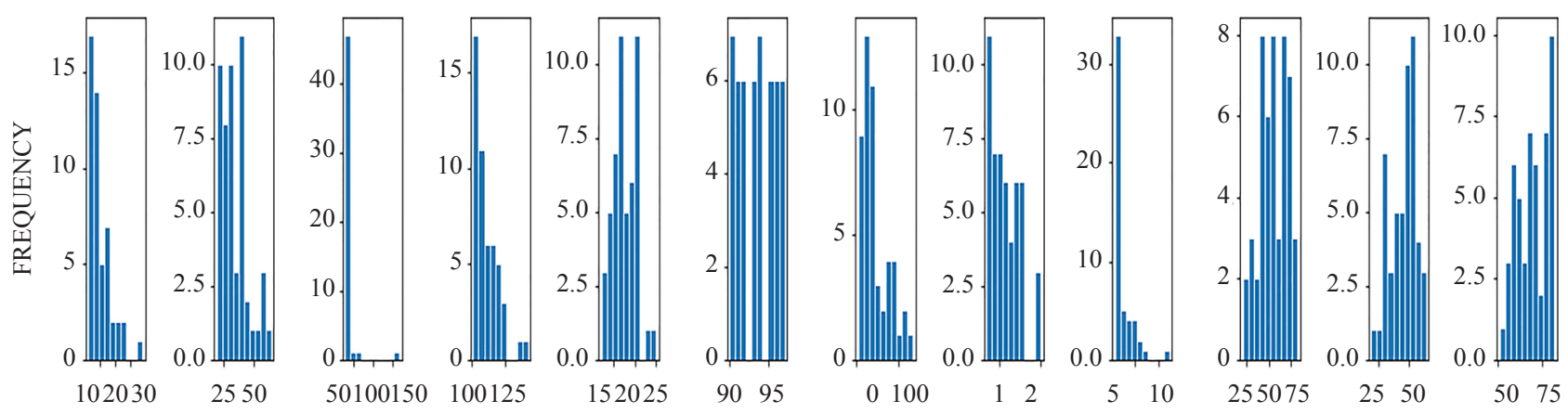

DURATION AGE BMI HR RR $\quad \mathrm{SpO}_{2}$

pNN50

LF/HF SD1/SD2

RMSSD SDNN FEV $_{1}$

Figure 2. Histogram plots of all factors for a) females and b) males 
b) None of the factors have Gaussian distributions, i.e., normally distributed. Kolmogorov-Smirnov test has also been conducted to test the normality check variable-wise for males and females (see Table 3).

Table 3. Results of Kolmogorov-Smirnov tests

\begin{tabular}{|c|c|c|c|c|c|c|c|c|c|c|c|c|}
\hline & DURATION & AGE & BMI & HR & $\mathrm{RR}$ & $\mathrm{SpO}_{2}$ & pNN50 & $\mathrm{LF} / \mathrm{HF}$ & $\begin{array}{l}\text { SD1/ } \\
\text { SD2 }\end{array}$ & RMSSD & SDNN & $\mathrm{FEV}_{1}$ \\
\hline Male & $\begin{array}{c}\text { KST: } \\
0.0657 ; \\
\text { p: } 0.1165 ; \\
\text { Skewness: } \\
\text { 1.6011; Kur- } \\
\text { tosis: } 3.2235\end{array}$ & $\begin{array}{c}\text { KST: } \\
0.14 ; \mathrm{p}: \\
0.25 ; \\
\text { Skew- } \\
\text { ness: } \\
0.444 \\
\text { Kurto- } \\
\text { sis:-0.396 }\end{array}$ & $\begin{array}{c}\text { KST: } \\
0.33 \text {; p: } \\
0.09 \text {; } \\
\text { Skew- } \\
\text { ness: } \\
4.194 \\
\text { Kur- } \\
\text { tosis: } \\
27.106\end{array}$ & $\begin{array}{c}\text { KST: } \\
0.476 ; \\
\text { p: } 0.67 \text {; } \\
\text { Skew- } \\
\text { ness: } \\
1.894 \\
\text { Kur- } \\
\text { tosis: } \\
9.396\end{array}$ & $\begin{array}{c}\text { KST: } \\
0.776 ; \\
\text { p: } 0.07 \text {; } \\
\text { Skew- } \\
\text { ness: } \\
2.894 \\
\text { Kur- } \\
\text { tosis: } \\
3.396\end{array}$ & $\begin{array}{c}\text { KST: } \\
0.476 ; \\
\text { p: } 0.17 \text {; } \\
\text { Skew- } \\
\text { ness: } \\
2.894 \\
\text { Kur- } \\
\text { tosis: } \\
12.396\end{array}$ & $\begin{array}{c}\text { KST: } \\
0.706 ; \mathrm{p}: \\
0.347 ; \\
\text { Skew- } \\
\text { ness: } \\
1.094 \\
\text { Kurto- } \\
\text { sis: } 2.6\end{array}$ & $\begin{array}{c}\text { KST: } \\
0.506 ; \mathrm{p}: \\
0.317 ; \\
\text { Skew- } \\
\text { ness: } \\
1.094 \\
\text { Kur- } \\
\text { tosis: } \\
1.396\end{array}$ & $\begin{array}{c}\text { KST: } \\
0.576 ; \mathrm{p}: \\
0.323 ; \\
\text { Skew- } \\
\text { ness: } \\
1.604 \\
\text { Kur- } \\
\text { tosis: } \\
2.326\end{array}$ & $\begin{array}{c}\text { KST: } \\
0.176 ; \\
\text { p: } 0.27 \text {; } \\
\text { Skew- } \\
\text { ness: } \\
1.19 \\
\text { Kur- } \\
\text { tosis: } \\
1.396\end{array}$ & $\begin{array}{c}\text { KST: } \\
0.176 ; \\
\text { p: } 0.13 \text {; } \\
\text { Skew- } \\
\text { ness: } \\
1.094 \\
\text { Kur- } \\
\text { tosis: } \\
5.396\end{array}$ & $\begin{array}{c}\text { KST: } \\
0.976 ; \\
\text { p: } 0.33 \text {; } \\
\text { Skew- } \\
\text { ness: } \\
1.94 \\
\text { Kurto- } \\
\text { sis: } 5.91\end{array}$ \\
\hline $\begin{array}{l}\mathrm{Fe}- \\
\text { male }\end{array}$ & $\begin{array}{l}\text { KST: } 0.145 ; \\
\text { p: } 0.215 ; \\
\text { Skewness: } \\
\text { 1.611; Kur- } \\
\text { tosis: } 3.105\end{array}$ & $\begin{array}{c}\text { KST: } \\
0.214 ; \mathrm{p}: \\
0.205 ; \\
\text { Skew- } \\
\text { ness: } \\
0.644 \\
\text { Kurto- } \\
\text { sis:-0.596 }\end{array}$ & $\begin{array}{c}\text { KST: } \\
0.23 \text {; p: } \\
0.07 ; \\
\text { Skew- } \\
\text { ness: } \\
4.894 \\
\text { Kur- } \\
\text { tosis: } \\
29.396\end{array}$ & $\begin{array}{c}\text { KST: } \\
0.376 ; \\
\text { p: } 0.37 ; \\
\text { Skew- } \\
\text { ness: } \\
1.894 \\
\text { Kur- } \\
\text { tosis: } \\
9.396\end{array}$ & $\begin{array}{c}\text { KST: } \\
0.376 ; \mathrm{p}: \\
0.217 ; \\
\text { Skew- } \\
\text { ness: } \\
2.094 \\
\text { Kur- } \\
\text { tosis: } \\
7.2396\end{array}$ & $\begin{array}{c}\text { KST: } \\
0.76 ; \mathrm{p}: \\
0.12 ; \\
\text { Skew- } \\
\text { ness: } 2.4 \\
\text { Kur- } \\
\text { tosis: } \\
10.396\end{array}$ & $\begin{array}{c}\text { KST: } \\
0.686 ; \mathrm{p}: \\
0.357 ; \\
\text { Skew- } \\
\text { ness: } \\
1.294 \\
\text { Kur- } \\
\text { tosis: } \\
2.234\end{array}$ & $\begin{array}{c}\text { KST: } \\
0.459 ; \\
\text { p: } 0.117 ; \\
\text { Skew- } \\
\text { ness: } \\
1.994 \\
\text { Kur- } \\
\text { tosis: } \\
1.996\end{array}$ & $\begin{array}{c}\text { KST: } \\
0.766 ; \mathrm{p}: \\
0.223 ; \\
\text { Skew- } \\
\text { ness: } \\
1.204 \\
\text { Kur- } \\
\text { tosis: } \\
3.326\end{array}$ & $\begin{array}{c}\text { KST: } \\
0.876 ; \\
\text { p: } 0.87 \text {; } \\
\text { Skew- } \\
\text { ness: } \\
1.39 \\
\text { Kur- } \\
\text { tosis: } \\
2.096\end{array}$ & $\begin{array}{c}\text { KST: } \\
0.179 ; \mathrm{p}: \\
0.103 ; \\
\text { Skew- } \\
\text { ness: } \\
1.994 \\
\text { Kurto- } \\
\text { sis: } 4.96\end{array}$ & $\begin{array}{c}\text { KST: } \\
0.887 \\
\text { p: } 0.38 \text {; } \\
\text { Skew- } \\
\text { ness: } \\
1.991 \\
\text { Kur- } \\
\text { tosis: } \\
5.881\end{array}$ \\
\hline
\end{tabular}

From the above table, it is evident that the variable 'duration of illness' is not much far from that which is normally distributed in males. The data shows a positive skewness and the Kurtosis value $>3$ signifies that the data has a heavier tail than that of the normal probability distribution. a p-value of each variable is $>0.05$ for both the males and females, corroborating the fact that the variables are not normally distributed.

c) Descriptive statistics (Tables 4 and 5), where cells containing abnormal values are marked by magenta color as the ready reckoner.

Table 4. Descriptive statistics of all the factors for females

\begin{tabular}{ccccccccc}
\hline & count & mean & std & min & $25 \%$ & $50 \%$ & $75 \%$ & max \\
\hline DURATION & 50 & 11.02 & 9.18 & 1 & 2.25 & 9 & 17.75 & 33 \\
AGE & 50 & 43.34 & 13.07 & 24 & 32 & 44.5 & 52 & 78 \\
BMI & 50 & 31.92 & 8.86 & 23.8 & 28 & 30.45 & 32.97 & 86.1 \\
HR & 50 & 111.84 & 10.29 & 101 & 103 & 108.5 & 116.5 & 138 \\
RR & 50 & 18.26 & 3.74 & 12 & 15 & 17.5 & 21 & 25 \\
SpO 2 & 50 & 92.32 & 2.16 & 90 & 91 & 92 & 93.75 & 97 \\
pNN50 & 50 & 38.96 & 28.72 & 9 & 20 & 33 & 41.75 & 116 \\
LF/HF & 50 & 1.224 & 0.4 & 0.6 & 0.925 & 1.2 & 1.47 & 2.3 \\
SD1/SD2 & 50 & 2.74 & 4.34 & 0.37 & 1.0125 & 1.475 & 2.9 & 29.81 \\
RMSSD & 50 & 61.43 & 17.47 & 25.7 & 47.5 & 60 & 73.65 & 97.4 \\
SDNN & 50 & 46.64 & 8.42 & 24.9 & 41.425 & 48.85 & 53.07 & 58.3 \\
FEV & 50 & 71.34 & 9.21 & 56 & 62.5 & 73 & 80 & 82 \\
\hline
\end{tabular}


Table 5. Descriptive statistics of all the factors for males

\begin{tabular}{|c|c|c|c|c|c|c|c|c|}
\hline & count & mean & std & $\min$ & $25 \%$ & $50 \%$ & $75 \%$ & $\max$ \\
\hline DURATION & 50 & 9.84 & 7.48 & 2 & 5 & 7.5 & 13 & 38 \\
\hline AGE & 50 & 33.94 & 10.75 & 19 & 25 & 31 & 39 & 65 \\
\hline BMI & 50 & 38.22 & 19.24 & 28.7 & 32.32 & 35.3 & 37.57 & 166.7 \\
\hline HR & 50 & 110.78 & 8.61 & 101 & 104 & 109 & 116 & 142 \\
\hline $\mathrm{RR}$ & 50 & 17.36 & 2.78 & 12 & 15 & 17 & 19.75 & 25 \\
\hline $\mathrm{SpO}_{2}$ & 50 & 93.44 & 2.32 & 90 & 91.25 & 93.5 & 95 & 97 \\
\hline pNN50 & 50 & 42.76 & 32.46 & 4 & 18.25 & 31.5 & 56.75 & 134 \\
\hline $\mathrm{LF} / \mathrm{HF}$ & 50 & 1.152 & 0.34 & 0.7 & 0.9 & 1.05 & 1.4 & 2 \\
\hline $\mathrm{SD} 1 / \mathrm{SD} 2$ & 50 & 2.265 & 2.17 & 0.71 & 1.005 & 1.305 & 2.4875 & 12.24 \\
\hline RMSSD & 50 & 54.9 & 14.84 & 21.4 & 44 & 55.25 & 67 & 82.1 \\
\hline SDNN & 50 & 44.86 & 10.1 & 19.4 & 38.25 & 46.9 & 51.75 & 63.3 \\
\hline $\mathrm{FEV}_{1}$ & 50 & 69.54 & 8.48 & 51 & 62.25 & 69.5 & 77 & 82 \\
\hline
\end{tabular}

d) $P C$ of all factors can be found in Table 6 (females) and Table 7 (males).

Table 6. Factor-wise PC results for females

\begin{tabular}{|c|c|c|c|c|c|c|c|c|c|c|c|c|}
\hline & DURATION & AGE & BMI & HR & $\mathrm{RR}$ & $\mathrm{SpO}_{2}$ & pNN50 & $\mathrm{LF} / \mathrm{HF}$ & $\mathrm{SD} 1 / \mathrm{SD} 2$ & RMSSD & SDNN & $\mathrm{FEV}_{1}$ \\
\hline DURATION & 1.00 & 0.71 & 0.07 & -0.03 & 0.61 & -0.07 & -0.10 & -0.04 & 0.03 & 0.20 & 0.21 & -0.76 \\
\hline AGE & 0.71 & 1.00 & 0.02 & -0.04 & 0.67 & 0.00 & -0.14 & -0.03 & 0.10 & 0.25 & 0.17 & -0.54 \\
\hline BMI & 0.07 & 0.02 & 1.00 & -0.28 & 0.18 & 0.05 & -0.05 & -0.08 & 0.16 & -0.06 & 0.06 & -0.12 \\
\hline HR & -0.03 & -0.04 & -0.28 & 1.00 & -0.08 & -0.03 & 0.00 & 0.07 & -0.06 & -0.05 & -0.07 & 0.17 \\
\hline RR & 0.61 & 0.67 & 0.18 & -0.08 & 1.00 & -0.02 & -0.21 & -0.08 & 0.34 & 0.10 & 0.06 & -0.49 \\
\hline $\mathrm{SpO}_{2}$ & -0.07 & 0.00 & 0.05 & -0.03 & -0.02 & 1.00 & -0.06 & -0.06 & 0.05 & 0.04 & 0.09 & 0.04 \\
\hline pNN50 & -0.10 & -0.14 & -0.05 & 0.00 & -0.21 & -0.06 & 1.00 & -0.14 & -0.10 & -0.13 & -0.05 & -0.03 \\
\hline $\mathrm{LF} / \mathrm{HF}$ & -0.04 & -0.03 & -0.08 & 0.07 & -0.08 & -0.06 & -0.14 & 1.00 & 0.09 & 0.49 & 0.44 & 0.08 \\
\hline $\mathrm{SD} 1 / \mathrm{SD} 2$ & 0.03 & 0.10 & 0.16 & -0.06 & 0.34 & 0.05 & -0.10 & 0.09 & 1.00 & -0.03 & -0.08 & -0.11 \\
\hline RMSSD & 0.20 & 0.25 & -0.06 & -0.05 & 0.10 & 0.04 & -0.13 & 0.49 & -0.03 & 1.00 & 0.86 & -0.07 \\
\hline SDNN & 0.21 & 0.17 & 0.06 & -0.07 & 0.06 & 0.09 & -0.05 & 0.44 & -0.08 & 0.86 & 1.00 & -0.11 \\
\hline $\mathrm{FEV}_{1}$ & -0.76 & -0.54 & -0.12 & 0.17 & -0.49 & 0.04 & -0.03 & 0.08 & -0.11 & -0.07 & -0.11 & 1.00 \\
\hline
\end{tabular}


Table 7. Factor-wise PC results for males

\begin{tabular}{|c|c|c|c|c|c|c|c|c|c|c|c|c|}
\hline & DURATION & AGE & BMI & $\mathrm{HR}$ & $\mathrm{RR}$ & $\mathrm{SpO}_{2}$ & pNN50 & $\mathrm{LF} / \mathrm{HF}$ & $\mathrm{SD} 1 / \mathrm{SD} 2$ & RMSSD & SDNN & $\mathrm{FEV}_{1}$ \\
\hline DURATION & 1.00 & 0.65 & -0.08 & -0.06 & 0.77 & -0.10 & 0.04 & 0.02 & -0.12 & 0.11 & 0.01 & 0.23 \\
\hline AGE & 0.65 & 1.00 & 0.12 & -0.19 & 0.53 & -0.11 & 0.11 & 0.00 & 0.05 & 0.22 & 0.10 & -0.10 \\
\hline BMI & -0.08 & 0.12 & 1.00 & -0.15 & -0.08 & -0.12 & -0.13 & -0.03 & 0.19 & -0.10 & -0.06 & -0.14 \\
\hline HR & -0.06 & -0.19 & -0.15 & 1.00 & 0.01 & 0.13 & -0.04 & -0.03 & -0.21 & 0.16 & 0.23 & 0.17 \\
\hline $\mathrm{RR}$ & 0.77 & 0.53 & -0.08 & 0.01 & 1.00 & -0.04 & -0.08 & -0.15 & -0.08 & -0.04 & -0.09 & 0.33 \\
\hline $\mathrm{SpO}_{2}$ & -0.10 & -0.11 & -0.12 & 0.13 & -0.04 & 1.00 & 0.00 & -0.30 & -0.09 & -0.02 & 0.03 & 0.07 \\
\hline pNN50 & 0.04 & 0.11 & -0.13 & -0.04 & -0.08 & 0.00 & 1.00 & -0.13 & 0.24 & 0.46 & 0.44 & 0.00 \\
\hline $\mathrm{LF} / \mathrm{HF}$ & 0.02 & 0.00 & -0.03 & -0.03 & -0.15 & -0.30 & -0.13 & 1.00 & 0.03 & 0.11 & -0.03 & -0.42 \\
\hline $\mathrm{SD} 1 / \mathrm{SD} 2$ & -0.12 & 0.05 & 0.19 & -0.21 & -0.08 & -0.09 & 0.24 & 0.03 & 1.00 & 0.23 & 0.13 & 0.06 \\
\hline RMSSD & 0.11 & 0.22 & -0.10 & 0.16 & -0.04 & -0.02 & 0.46 & 0.11 & 0.23 & 1.00 & 0.91 & 0.05 \\
\hline SDNN & 0.01 & 0.10 & -0.06 & 0.23 & -0.09 & 0.03 & 0.44 & -0.03 & 0.13 & 0.91 & 1.00 & 0.07 \\
\hline $\mathrm{FEV}_{1}$ & 0.23 & -0.10 & -0.14 & 0.17 & 0.33 & 0.07 & 0.00 & -0.42 & 0.06 & 0.05 & 0.07 & 1.00 \\
\hline
\end{tabular}

f) Linear regression plots for females and males are visible in Figures 3 and 4, respectively.
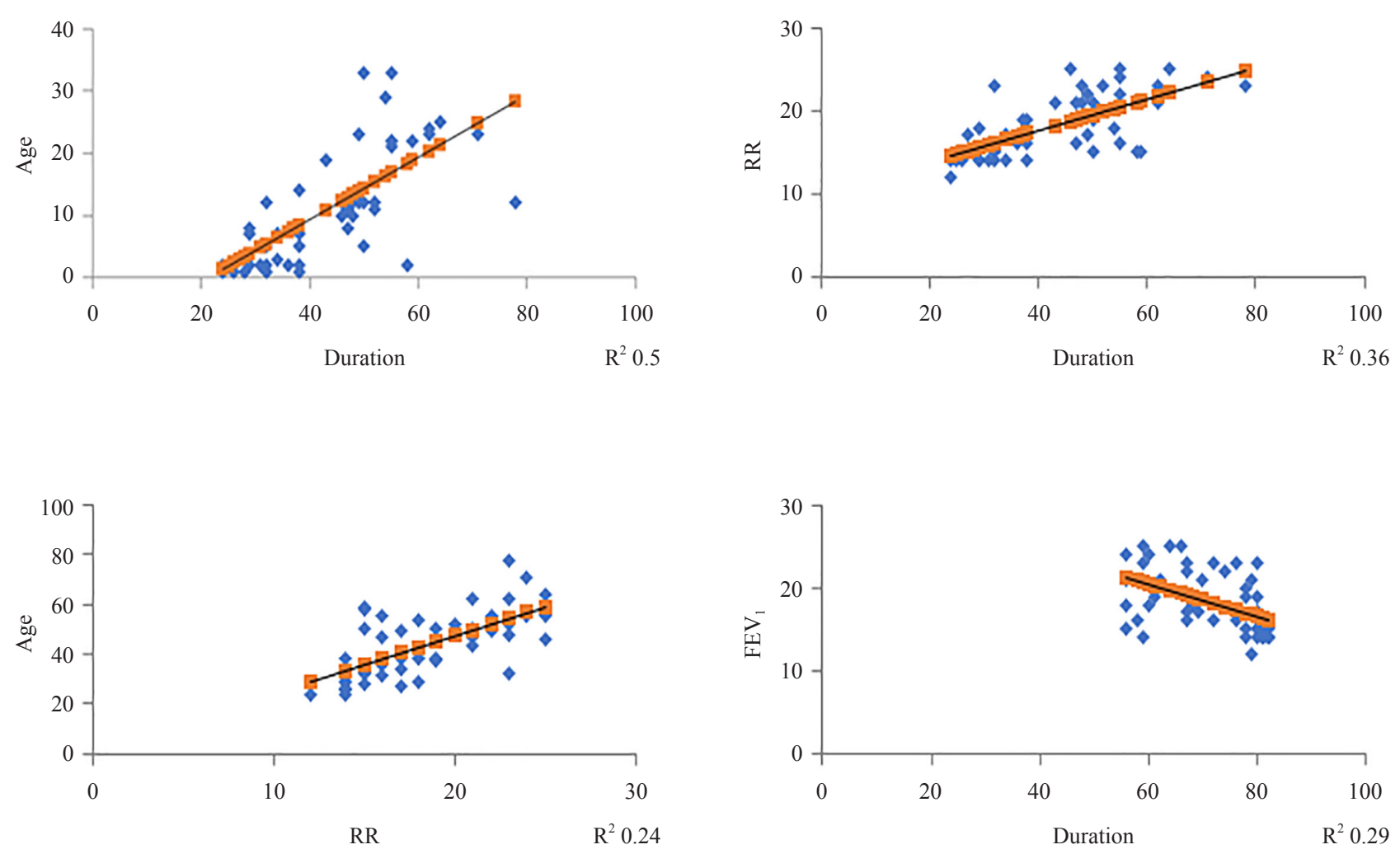

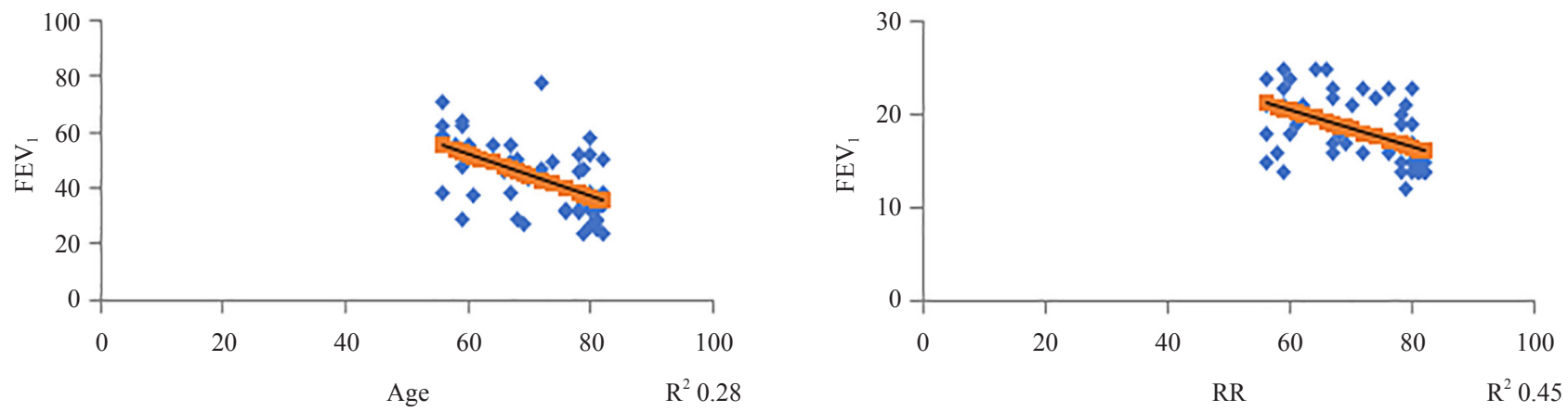

Figure 3. Regression plots of females
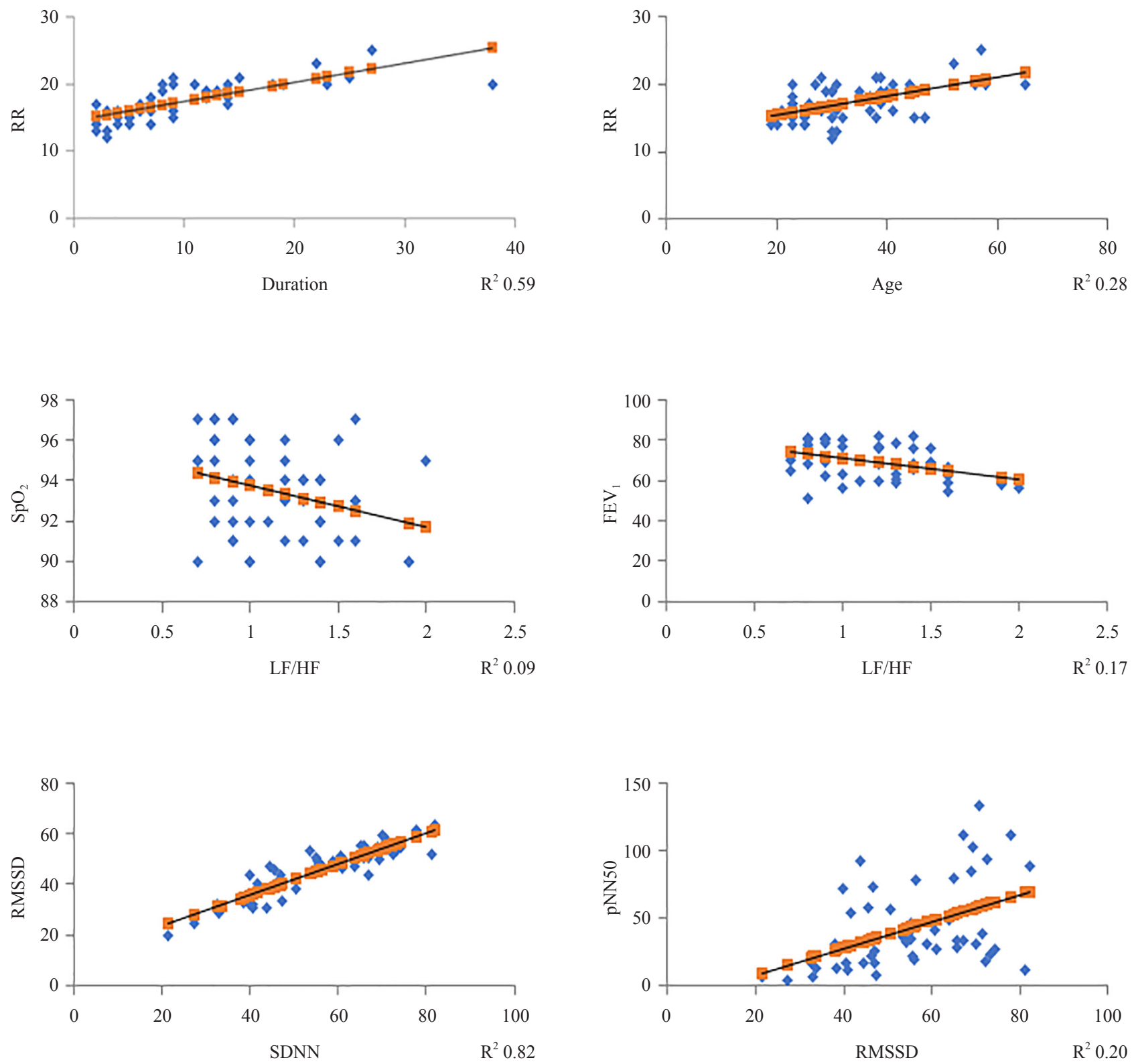


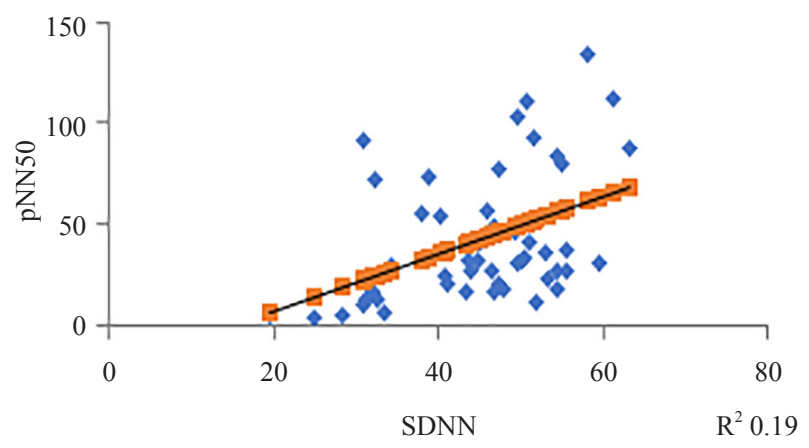

Figure 4. Regression plots of males

\section{Discussions}

This section discusses the findings of the experiments one by one.

The distributions of data (Box plots, Figures $2 a$ and 2b, and Table 3 ) are non-normal for all the attributes, as none of these could fit in a bell-shaped curve. Clinical data are usually non-normal due to the psychophysiological differences among the individuals [21].

Table 8. The overview of the PC results for the sample

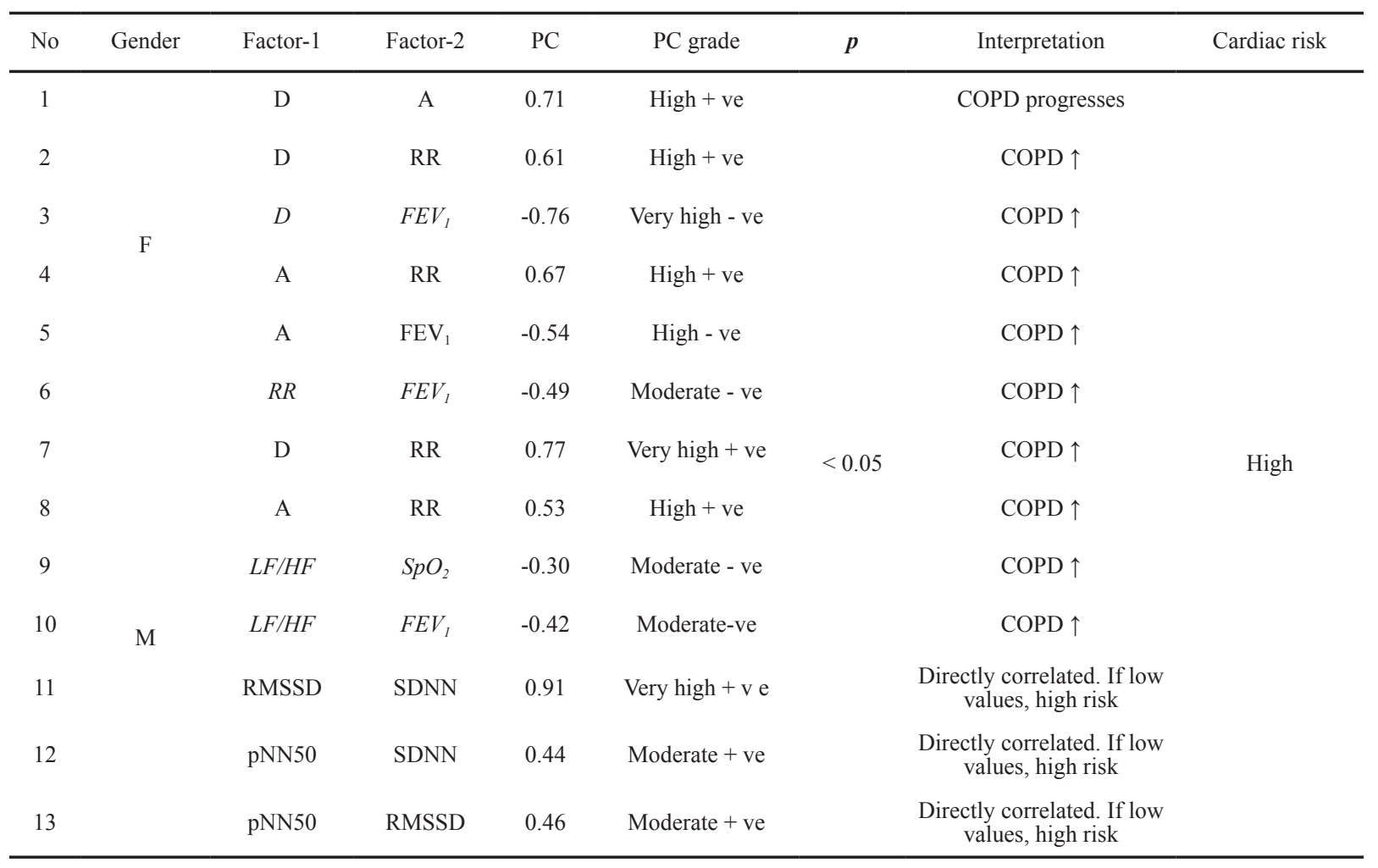

Descriptive statistics (Tables 4 and 5) provide the skeleton of a dataset. From mean, median (50 ${ }^{\text {th }}$ Quartile), and standard deviation, data concentration, and its dispersions can be well-noted in a sample. In this study, both the 
sexes are obese (mean BMI > 30), have tachycardia (mean HR > 100), both indicate higher cardiovascular risk [22] [23], respectively. Interestingly, in females, the mean SD1/SD2 > 2.5, indicating higher cardiovascular risks [24]. It is important to note that as the age progress over 12 years, females have higher HR than males, which is also evident from the Lyfas HR values (mean $111.84 \mathrm{bpm}$ in females and $110.78 \mathrm{bpm}$ in males) [14].

$P C$ results of females $(\mathrm{F})$ and males $(\mathrm{M})$ can be seen in Table 8. Negative PC scores are italicized.

The $R^{2}$ values of the predictions are found to be lower than 0.5 in this work. It is important to mention here that the low value does not mean that the model is poor [25]. Such lower values may occur due to unexplainable variations of the factors, when captured, which could be due to the variations in the CVAM that varies from one individual to another individual, according to the authors. However, from the p-values $(<0.0336)$, it is evident that the model is statistically significant.

It is important to mention that PC show weaker correlations between each of the digital cardiovascular biomarkers and $\mathrm{FEV}_{1}$, except for the LF/HF. The reason for the weak correlation could be because the "parasympathetic compensatory mechanism' is good in most cases as the body attempts to adapt to the pathophysiological changes of COPD over a certain period of the disease.

The cascading interplay of the factors can be seen in Figure 5.

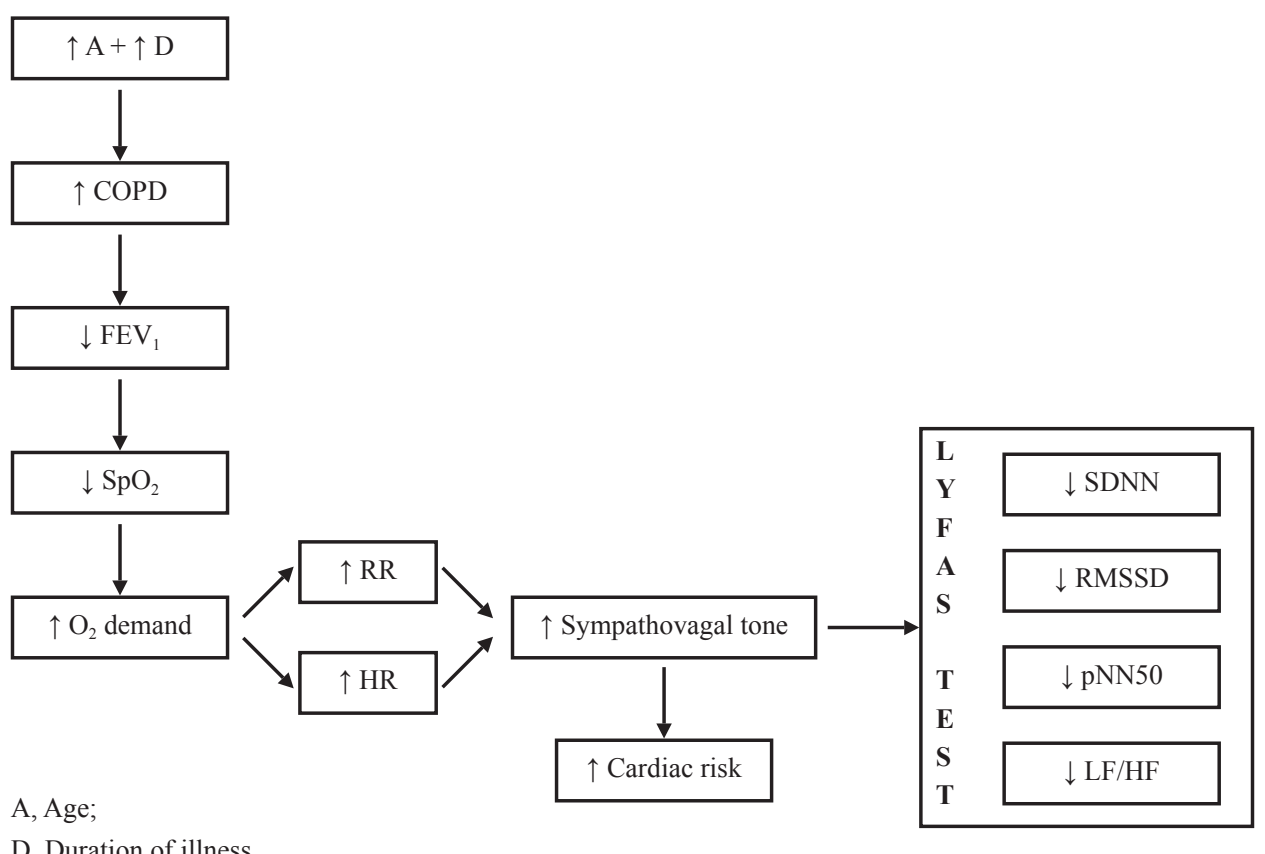

Figure 5. Cardiac risk monitoring of COPD using Lyfas

In the above Figure, it is evident that the RMSSD and SDNN have a 'very high' inter-class positive correlation as high values of both refer to the parasympathetic balance of the body. Increased Age (A) and Duration of COPD (D) as progress, gradually the illness worsens irrespective of the treatment the patient receives [26], which is evident by lowered $\mathrm{FEV}_{1}$ scores [27]. However, low positive correlations between D-RMSSD and D-SDNN could be the fact that the body attempts to adapt to the pathophysiological changes for a certain period irrespective of the speed of worsening of the illness. In chronic hypoxia, parasympathetic overdrive has been noted to reduce the HR at a high altitude is one good example to support the fact [28]. Moderate negative correlations between LF/HF-FEV ${ }_{1}$ and $\mathrm{LF}^{\prime} \mathrm{HF}_{-} \mathrm{SpO}_{2}$ is an interesting finding, which signifies the fact that $\mathrm{FEV}_{1}$ and $\mathrm{O}_{2}$ saturation physiologically modulates the sympathovagal balance. A high sympathetic drive produces hypoxia and hence reduces $\mathrm{FEV}_{1}$ and vice versa, which means LF/HF behaves as a confounding variable that influences both the independent variable $\mathrm{SpO}_{2}(p$-value 0.0333) and dependent 
variable $\mathrm{FEV}_{1}$ (p-value 0.0021$)$ in males. It potentially corroborates the fact that digital biomarkers relate to clinical indicators, measured with a dedicated biomedical instrument, called Lyfas. An ever-increasing $\mathrm{O}_{2}$ demand in the body can also be assessed through the lower $\mathrm{SpO}_{2} \%$. As a result, $\mathrm{HR}$ and $\mathrm{RR}$ are increased, and for that, a higher sympathetic drive is needed. As mentioned earlier, that, the signature of high sympathetic drive in mind-body homeostasis to meet up the increasing $\mathrm{O}_{2}$ demand of the body can be captured efficiently from the peripheral pulse of index fingers using Lyfas and its heuristics-based analytics can evaluate such sympathetic overdrive by examining the relevant HRVcorrelates, such as SDNN, RMSSD, LF/HF, pNN50, and SD1/SD2. In this study, SDNN, RMSSD, pNN50, and LF/ HF are found to be statistically significant (p-values SDNN-RMSSD: 1.34E-19; RMSSD-pNN50: 0.000898; SDNNpNN50: 0.001264; LF'HF-RMSSD: 0.00012; LF/HF-SDNN: 0.00010; Lf/HF-pNN50: 0.0000912 ) in determining the impending cardiac risk in COPD patients using Lyfas.

\section{Conclusions}

The study concludes that,

- Lyfas is not a hypersensitive device to assess the mind-body homeostasis by measuring the optical biomarkers that surrogate for the CVAM. Therefore, it is clinically reliable.

- Naturally, the clinical data may not be normally distributed due to the inherent physiological variations of the individuals irrespective of age and gender, although it is important to test to choose the correct statistical methods for further analysis.

- Lyfas captures the cardiovascular digital biomarkers referring to the sympathovagal balance, such as low (SDNN, $p N N 50, R M S S D)$, and high $(L F / H F)$ those are statistically significant in monitoring the imminent cardiac risk in COPD cases. $\mathrm{LF} / \mathrm{HF}$ is a confounding variable, when increased, $\mathrm{SpO}_{2}$ and $\mathrm{FEV}_{1}$ decrease indicating the potential cardiopulmonary risk in the patient.

- Lyfas can be a useful instrument as the patients can take the tests at home from their smartphones and share the report with their doctors with the help of mobile data or wifi for further medical guidance. The effectiveness of Lyfas would be the most advantageous if used under the guidance of a medical doctor who is either a pulmonologist, or a specialist in internal medicine, or a cardiologist, or an anesthesiologist. Another advantage of Lyfas is that the doctor can also monitor the patients seamlessly sitting away from them. Such a ubiquitous property of Lyfas may reduce the chance of acute hospitalization and death.

- The frequency of Lyfas testing depends on the severity of the illness. Based on the observations, authors recommend that Lyfas can be tested monthly once for mild to moderate grades of cases $\left(\mathrm{SpO}_{2} 88-92 \%\right.$, RR less than 20 per minute, $\mathrm{FEV}_{1} 50-80 \%$ ), and weekly in moderate to severe grades of COPDs alongside other physiological parameters, such as $\mathrm{SpO}_{2}$ (below 88\%), RR (above 20 per minute), and $\mathrm{FEV}_{1}$ (equal to or below 50\%) or at a timely interval as per the advice of the medical doctors. LF/HF greater than 2 poses a suspected cardiac risk and values greater than or equal to 2.5 confirm the cardiac risk by Lyfas. It could be useful for the therapeutic and prognostic assessment of any COPD case pervasively, especially for the elderly population who can not travel to the hospital with low $\mathrm{FEV}_{1}$ and $\mathrm{SpO}_{2}$.

- High LF/HF, low RMSSD, low SDNN, low pNN50, and high SD1/SD2 are reliable biomarkers of the cardiac risk in place of $\mathrm{SpO}_{2}$ and $\mathrm{FEV}_{1}$.

- The pervasive, non-invasive, analytics-enabled, personalized monitoring of cardiac risk by Lyfas would always be an advantage as a smartphone-based home-healthcare biomarker instrument under the expert guidance of medical doctors.

\section{Acknowledgement}

TIDE 2.0 Program, DERBI Foundation, The Ministry of Electronics and Information Technologies, Govt. of Karnataka, India. Certificate number: IN-KA45186921153022T, Dated: 13th July 2021. 


\section{Registration of the clinical study}

The study protocol was approved (No. ECR/1181/Inst/K.A., 2019, dated 30th January 2020) by the external ethical committee, namely Vagas Institutional Ethics Committee review board, India.

\section{Conflict of interest}

The authors declare that there is no personal or organizational conflict of interest with this work.

\section{References}

[1] Telugunta R, Gill S, Sumant O. Mobile health market overview. 2020. Available from: https://www. alliedmarketresearch.com/mobile-health-market [Accessed 11th November 2021].

[2] Rajeswari J, Jagannath M. Advances in biomedical signal and image processing-A systematic review. Informatics in Medicine Unlocked. 2017; 8: 13-19. Available from: https://doi.org/10.1016/j.imu.2017.04.002.

[3] Deepa HS, Das R. Evaluation of non-invasive smartphone based digital biomarker tool lyfas in detecting sleep deficiency and its effects: A retrospective observational study. Indian Journal of Applied Research. 2021; 11(1): 4647.

[4] Das R, Chattopadhyay S. Towards cardiac risk monitoring of duchene muscular dystrophy using lyfas. Journal of Nanotechnology in Diagnosis and Treatment. 2021; 7: 25-32. Available from: http://dx.doi.org/10.12974/23118792.2021.07.3.

[5] World Health Organization. Chronic obstructive pulmonary disease (COPD). 2021. Available from: https://www. who.int/news-room/fact-sheets/detail/chronic-obstructive-pulmonary-disease-(copd) [Accessed 11th November $2021]$.

[6] Garrison DM, Pendela VS, Memon J. Cor pulmonale. StatPearls [Internet]. Treasure Island (FL): StatPearls Publishing; 2021.

[7] Leong P, MacDonald MI, King PT, Osadnik CR, Ko BS, Landry SA, et al. Treatable cardiac disease in hospitalised COPD exacerbations. ERJ Open Research. 2021; 7(1): 756-2020.

[8] Al-Shura NA. Dyspnea and orthopnea. Inquiry, Treatment Principles and Plans in Integrative Cardiovascular Chinese Medicine. Academic Press; 2020. p. 43-51. Available from: https://doi.org/10.1016/C2018-0-02061-7.

[9] Clinic M. Heart Failure. 2021. Available from: https://www.mayoclinic.org/diseases-conditions/heart-failure/ symptoms-causes/syc-20373142 [Accessed 11th November 2021].

[10] Ching SM, Chia YC, Lentjes M, Luben R, Wareham N, Khaw KT. FEV 1 and total cardiovascular mortality and morbidity over an 18 years follow-up Population-Based Prospective EPC-NORFOLK Study. BMC Public Health. 2019; 19(1): 501. Available from: doi.org/10.1186/s12889-019-6818-X.

[11] Mathew KG, Aggaewal P. Diseases of cardiovascular system. New Delhi: RELX India Pvt. Ltd; 2019.

[12] Shaffer F, Ginsberg JP. An overview of heart rate variability metrics and norms. Frontiers in Public Health. 2017; 28(5): 258.

[13] Chattopadhyay S, Das R. Comparing heart rate variability with polar H10 sensor and pulse rate variability with LYFAS: A novel study. Journal of Biomedical Engineering and Technology. 2021; 9(1): 1-9.

[14] John Hopkins Medicine. 2021. Available from: https:/www.hopkinsmedicine.org/health/conditions-and-diseases/ vital-signs-body-temperature-pulse-rate-respiration-rate-blood-pressure\#: : text $=$ The $\% 20$ normal $\% 20$ pulse $\% 20$ for $\% 20$ healthy,heart $\% 20$ rates $\% 20$ than $\% 20$ do $\% 20$ males [Accessed 12 th November 2021].

[15] Oxygen Levels, Pulse Oximeters, and COVID-19. Minnesota department of health; 2021. Available from: https:// www.health.state.mn.us/diseases/coronavirus/pulseoximeter.html\#: :text=A\%20normal\%20level\%20of $\% 20$ oxygen,call\%20your\%20health\%20care\%20provider [Accessed 12th November 2021].

[16] Adult BMI Calculator. CDC; 2020. Available from: https://www.cdc.gov/healthyweight/assessing/bmi/adult_bmi/ english_bmi_calculator/bmi_calculator.html [Accessed 12th November 2021].

[17] Mathew KG, Aggaewal P. Diseases of the respiratory system. New Delhi: RELX; 2019.

[18] Goforth C. Using and interpreting cronbach's alpha. University of Virginia Library; 2015. Available from: https:// data.library.virginia.edu/using-and-interpreting-cronbachs-alpha/ [Accessed 11th January 2021].

[19] Stolarova M, Wolf C, Rinker T, Brielmann A. How to assess and compare inter-rater reliability, agreement and 
correlation of ratings: An exemplary analysis of mother-father and parent-teacher expressive vocabulary rating pairs. Frontiers in Psychology. 2014; 5: 509.

[20] Chattopadhyay S, Shinha P. Understanding factors impacting covid vaccination in India: A preliminary report. Quantun Journal of Medical and Health Sciences. 2021; 1(3): 18-31.

[21] Bono R, Blanca MJ, Arnau J, Gómez-Benito J. Non-normal distributions commonly used in health, education, and social sciences: A systematic review. Frontiers in Psychology. 2017; 8: 1602.

[22] Cercato C, Fonseca FA. Cardiovascular risk and obesity. Diabetology and Metabolic Syndrome. $2019 ; 11: 74$. Available from: https://doi.org/10.1186/s13098-019-0468-0.

[23] Cierpka-Kmieć K, Hering D. Tachycardia: The hidden cardiovascular risk factor in uncomplicated arterial hypertension. Cardiology Journal. 2020; 27(6): 857-867.

[24] Koichubekov B, Riklefs V, Sorokina M, Korshukov I, Turgunova L, Laryushina Y, et al. Informative nature and nonlinearity of lagged poincaré plots indices in analysis of heart rate variability. Entropy. 2017; 19(10): 523.

[25] Grace-Martin K. Can a regression model with a small R-squared be useful? The Analysis Factor. 2021. Available from: https://www.theanalysisfactor.com/small-r-squared/ [Accessed 16 November 2021].

[26] Mathioudakis AG, Vanfleteren LE, Lahousse L, Higham A, James GC, Allinson P, et al. Current developments and future directions in COPD. European Respiratory Review. 2020; 29(158): 200289.

[27] Agarwal AK, Raja A, Brown BD. Chronic obstructive pulmonary disease. StatPearls [Internet]. Treasure Island (FL): StatPearls Publishing; 2021.

[28] Boushel R, Calbet JAL, Rådegran G, Sondergaard H, Wagner PD, Saltin B. Parasympathetic neural activity accounts for the lowering of exercise heart rate at high altitude. Circulation. 2001; 104: 1785-1791. Available from: doi.org/10.1161/hc4001.097040. 